\title{
Normas españolas para la transcripción y edición de colecciones diplomáticas
}

\author{
José Miguel López Villalba*
}

\begin{abstract}
RESUMEN
El contenido de las fuentes antiguas, su transcripción y correspondiente edición ha llevado, ya desde el siglo xviII, a que los paleógrafos y diplomatistas hayan tenido una preocupación prioritaria en el establecimiento de normas que fijasen la publicación de las citadas fuentes con el máximo rigor.
\end{abstract}

En este trabajo hemos estudiado en sus diferentes aspectos las propuestas de normas españolas, comenzando por la evolución cronológica y el ámbito donde se fijaron, para continuar con el análisis pormenorizado de los aspectos más relevantes de las citadas normas: Formación y ordenación de las colecciones diplomáticas; Estructura de la edición y contenido de sus partes: fecha, resumen, aparato crítico, notas históricas y disertación crítica; El texto: normas para su transcripción; Presentación de la edición: cuerpo y

\section{ABSTRACT}

The content of the ancient sources, their transcription and their subsequent edition, has led paleographs and diplomatists, since the xvilth Century, to give a priority to fix the rules of the publication of the above mentioned sources with the maximum rigour. In this report we have studied in their differents aspects, the spanish rules proposals, starting with the evolution and environment where they were fixed, and continuing with a detailed analysis of the most relevant points of the mentioned rules: Formation and ordering of the diplomatic collections; Edition structure and content of its parts: date, summary, critycal apparatus, historic notes and critic dissertation; The text: Transcription rules; Edition appearance: Word typography and size, appendixes and indexes.

As a conclusion, we could underline that the singularity of the high number

* UNED. 
tamaño de la letra y, por último, apéndices e indices. Como conclusión destacariamos que el particularismo de las numerosas normas españolas pasa generalmente por detalles secundarios que, sin embargo, efectan al conjunto de la edición por lo que proponemos seguir

las normas de la Commision Internationale de Diplomatique. of the spanish rules is generally based in secondary details, although affecting the edition as a whole, so we propose to follow the rules of the International Diplomatique Commission.

\section{INTRODUCCIÓN}

La transcripción y edición de textos y documentos ha sido durante mucho tiempo objeto preferente de interés para amplios colectivos científicos, puesto que en su elaboración han participado prioritariamente historiadores, paleógrafos y diplomatistas, filólogos y documentalistas. Producto de esta laboriosa tarea es la existencia actual de una amplísima y variada tipología de fuentes que ilustra los campos más diversos del conocimiento humano y por tanto del saber. Naturalmente, es en este ámbito donde el tema relacionado con las normas, reglamentaciones o recomendaciones de transcripción y edición cobra su máximo interés, pudiéndose detectar ya a finales del siglo XVIII una preocupación por su establecimiento entre los paleógrafos de la época.

Ahora bien, la incomprensible ausencia de una diferenciación conceptual entre lo que podría constituir una simple transcripción-edición y un estudio o edición crítica de cualquier texto o documento ha originado la paradoja de poder encontrar en la actualidad un doble fenómeno: por una parte, la existencia de «normas de transcripción y edición» para historiadores, para filólogos ${ }^{1}$, para historiadores del derecho, para paleógrafos y diplomatistas, etc., contraviniendo lógicamente la esencia misma del sig-

1 Recientemente, el profesor P. SÁnCHEz-PRIETo Borja en su obra: Cómo editar los textos medievales. Criterios para su presentación gráfica. (1998), realiza una propuesta orgánica cuyos criterios resultan próximos, por familiaridad de contenidos a la actividad científica de la Paleografía. Dejando de lado la validez o certeza de alguno de ellos, llama aún más la atención su ejercicio conceptualizador de la crítica textual y, naturalmente el lugar reservado en el mismo a la Paleografia, a la que concibe como disciplina encargada exclusivamente de la lectura y transcripción de los testimonios manuscritos, enajenándole de todo un completo y fundamentado conjunto de intereses que ha sido perfectamente racionalizado en la ultima propuesta de edición de textos latinos de la Edad Media elaborada por la Commision Internationale de Diplomatique (1984), obra que curiosamente no cita el mencionado autor. 
nificado último que posee el término "norma»; $y$, por otra parte, la natural ausencia de diferenciación que poseen tales normas, participando a menudo de contenidos ajenos al análisis crítico de la información textual ${ }^{2}$.

A pesar de la importancia que reviste este hecho, no pretendemos arrogar la transcripción y edición de fuentes a los especialistas en Ciencias y Técnicas Historiográficas, sino más bien y convencidos del lugar que ocupa esta tarea en el quehacer científico de esta disciplina, nuestra intención se cifra en analizar la problemática que presenta su realización a la vista de las diferentes "normas" elaboradas por los más insignes paleógrafos y diplomatistas españoles.

A mediados de los años sesenta, el profesor Filemón Arribas Arranz ya trazó un breve, aunque acertado, panorama evolutivo de la normativa española para la transcripción de textos y documentos, señalando la existencia de dos corrientes bien diferenciadas. La primera, iniciada a finales del siglo XIX a través de la publicación de las grandes colecciones documentales y con una denotada finalidad histórica, vería su mayor hito en la normativa propuesta en 1944 por la Escuela de Estudios Medievales. Mientras que la segunda, caracterizada prioritariamente por un interés paleográfico, se encontraría representada por las normas de autores como Esteban de Terreros y Pando, Andrés Merino de Jesucristo, Jesús Muñoz y Rivero, Zacarías García Villada y, ya en pleno siglo xx, por las sistematizaciones realizadas por Agustín Millares Carlo ${ }^{3}$.

El análisis comparativo de las normas más relevantes de ambas corrientes - la normativa de 1944 y las del profesor Millares- permite apreciar algunas diferencias importantes que, posteriormente, se han venido manteniendo en los diferentes tratados españoles de Paleografía y en las colecciones diplomáticas más destacadas, existiendo en consecuencia una falta de acuerdo abiertamente reconocida por sus propios autores ${ }^{4}$.

2 Una bibliografía selectiva sobre las distintas normas de transcripción y edición de fuentes aparece recogida por Emilio SAEZ (+) y Carlos SAEZ, Colección diplomática del Monasterio de Celanova (842-1230), I. Universidad de Alcalá de Henares, 1996, pp. 19-33.

3 Arribas Arranz, Filemón, Paleografía documental hispánica. Universidad de Valladolid, 1965, pp. 9-17.

4 Vid. al respecto las declaraciones de A. Millares Carlo y J. M. Ruiz Asencio, Tratado de Paleografía Española. (3. ${ }^{\mathrm{a}}$ ed.), Madrid, Espasa-Calpe, 1983, p. XII.

También resultan significativas las precisiones que al respecto realizaron en los diferentes volúmenes a su cargo Emilio SAEZ, Colección documental del archivo de la catedral de León I (775. 1230), León, CSIC-CECEL. Caja de Ahorros y Monte de Piedad de León. Archivo Histórico Diocesano de León, 1987, p. LXX, y por otro lado Jose Manuel Ruiz AsEncio, Colección documental del archivo de la catedral de León III (775-1230), León, CSIC-CECEL. Caja de Ahorros y Monte de Piedad de León. Archivo Histórico Diocesano de León, 1987, p. XX 
El caso español no fue un hecho aislado, pudiéndose detectar asimismo esta falta de criterios comunes para la transcripción y edición de documentos en otros muchos países. Por esta razón, la Comisión Internacional de Diplomática inició en 1974 una serie de trabajos técnicos orientados a la elaboración de una normativa internacional de la que pudieran servirse todos los colectivos científicos, la cuál vió definitivamente la luz en $1984{ }^{5}$.

No obstante, el seguimiento de sus principios no se ha visto tan desarrollado como era de esperar, pudiéndose observar todavía el mantenimiento de posturas que siguen anteriores individualismos muy destacados así como defensores a ultranza de una total libertad ${ }^{6}$.

Las divergencias apreciadas en las normas españolas para la transcripción y edición de textos y documentos no cuentan con un análisis exhaustivo que permita, no tanto valorar la conveniencia de utilizar unas u otras, es decir, de establecer criterios de calidad, cuanto de estudiar la naturaleza misma de tales discrepancias, objetivo básico de este artículo. En otras palabras, las diferencias de opinión que separan a los distintos autores responsables de estas normativas ¿a qué se deben?, ¿en qué aspectos se diferencian?, ¿a qué obedecen tales diferencias en caso de existir?

Consideramos que se trata de un tema polémico pero no por ello marginal a los intereses más inmediatos de paleógrafos y diplomatistas, puesto que la valiosa tarea de edición de fuentes, no por antigua ha quedado obsoleta en nuestro quehacer científico.

Antes de iniciar nuestro análisis, estimamos necesario delimitar su campo de acción con objeto de precisar claramente los objetivos de este trabajo.

\section{Cronología y ámbito}

Ya que en este caso no pretendemos fijar la atención en un estudio evolutivo de la normativa elaborada para la transcripción y edición de fuen-

\footnotetext{
5 La primera edición de dicha normativa - Normalisation Internationale des methodes de publication des documents latins du Moyen Age. Roma, Commission Internationale de Diplomatique, 1977- fue objeto de observaciones complementarias posteriores en los coloquios técnicos celebrados en Madrid 1978, Roma 1979 y Zaragoza 1982, siendo publicada definitivamente dos años más tarde: Commission Internationale de Diplomatique, Commission Internationale de Sigillographie, Folia Caesaraugustana 1. Diplomatica et Sigillographica. Zaragoza, Institución «Fernando el Católico", 1984.

6 Dentro del ámbito español, el profesor Carlos Sáez se declara abiertamente partidario de esta última tendencia, siguiendo en este sentido las opiniones de investigadores italianos como $\mathrm{A}$. Bartoli o Silio P.P. Scalfati. Vid. al respecto E. SAEZ - C. SAEZ, op. cit., p. 22.
} 
tes documentales, centraremos nuestro análisis en las normas aparecidas en España a lo largo del siglo xx, arrancando de la propuesta por $Z$. García Villada por tratarse de la primera manifestación en la que el progreso de la metodología histórica aplicada a la transcripción de documentos se hizo efectivo.

\section{Autores y normativas}

Conscientes de la amplitud desarrollada en este campo a través de las múltiples "normativas" particulares de los diferentes autores dedicados a la transcripción y edición de fuentes documentales, hemos operado una selección basada en las normas que más seguimiento y aceptación han tenido a lo largo de toda la centuria, correspondiendo, como era de esperar, con los hitos más significativos de la Paleografía y Diplomática españolas. Este consensuado seguimiento es obra, por lo general, de la reconocida tarea de docencia y magisterio de sus autores, apareciendo muchas veces sus propuestas en obras de marcado carácter pedagógico, como manuales y tratados ?

\section{Naturaleza de las normativas seleccionadas}

La diferenciación observada en nuestras normativas en torno al tratamiento de las colecciones diplomáticas y los textos literarios ha hecho que centremos en esta ocasión nuestro interés en el análisis de las primeras, reservando para otra ocasión el estudio de los textos.

7 García Villada, Zacarías, Metodología y crítica históricas. (2." ed, Barcelona, Sucesores de Juan Gili, S.A., 1921). Barcelona, Ed. El Albir, 1977, cap. XVIII, pp. 301-309.

Normas de transcripción y edición de textos y documentos. Madrid, C.S.l.C., Escuela de Estudios Medievales (EEM), 1944. Según señala Concepción Mendo Carmona apoyándose en un estudio del profesor Eloy Benito Ruano, parece que fue Emilio Sáez el autor anónimo de estas normas. Vid. C. MENDO CARMONA, La Escuela de Estudios Medievales: su labor de edición de fuentes, Hispania, L/2, 175 (1990), p. 607, nota 32.

Millares CaRlo, Agustín y Mantecón, José Ignacio, Album de Paleografia Hispanoamericana de los siglos XVI Y XVII. México, 1955.

Abribas Arranz, Filemón, op. cit.

Millares Carlo, Agustín y Ruiz Asencio, José Manuel, op. cit.

SAEZ, Emilio, op. cit.

Ruiz Asencio, José Manuel, op. cit.

SAEZ, Emilio y SAEZ, Carlos, op. cit.

Folia Caesaraugustana I, op. cit.

Por razones de concisión, las citas que seguirán a partir de este momento sobre las mencionadas normativas se realizarán utilizando simplemente el año de su publicación. 


\section{ANÁLISIS}

Los aspectos que serán objeto de análisis en las diferentes normas elaboradas para la edición y transcripción de documentos son los siguientes:

1. Formación y ordenación de colecciones diplomáticas.

2. Estructura de la edición y contenido de sus partes: fecha, resumen, aparato crítico, notas históricas y disertación crítica.

3. El texto: normas para su transcripción.

4. Presentación de la edición: cuerpo y tamaño de la letra.

5. Apéndices e índices.

\section{Formación y ordenación de colecciones diplomáticas}

Varios son los puntos de interés que incluye el análisis de este primer apartado.

En primer lugar la postura ante los criterios de formación de colecciones diplomáticas, aspecto sobre el cual encontramos tan sólo una declaración expresa en la normativa del C.S.I.C., en la que claramente se citan tres criterios distintos: la personalidad del otorgante, la entidad que los recibe y los referentes a determinados hechos o instituciones ${ }^{8}$.

En cuanto al grado o nivel de atención que requiere la edición de documentos de una colección diplomática según su cronología, sí se ha encontrado diversidad de criterios. La postura de Z. García Villada será continuada con ligeras variantes por la normativa del C.S.I.C., puesto que en ambas se defiende la extremada escrupulosidad que debe regir cualquier edición de documentos anteriores a mediados del siglo XIII, los cuales deberán ser incluidos íntegramente, recibiendo los posteriores a esa fecha idéntico tratamiento sólo cuando ofrezcan el suficiente interés por su contenido o por su forma; del resto de los documentos se proporcionará una simple ficha catalográfica en la que se dejará constancia de la parte dispositiva o de aquella que se estime más interesante ${ }^{9}$.

No parecen mantener idéntico criterio A. Millares y José I. Mantecón, pues conscientes de la falta de rigor a la hora de transcribir fuentes docu-

1944, n. ${ }^{\circ} 106$, p. 33.

1921, p. 308.

1944, n. 109, p. 34. 
mentales correspondientes a la Edad Moderna, propugnan que "como todos los demás documentos históricos, para ser utilizados como fuente segura de conocimiento, deben ser presentados al investigador en tal forma que reproduzcan exactamente su contenido y con las características formales propias de su época” ${ }^{10}$.

Sin embargo, todos los autores convienen en asignar una numeración arábiga correlativa a los distintos documentos que sean objeto de edición y transcripción, así como en establecer un criterio cronológico en la ordenación de los mismos.

A este respecto, cabe resaltar que la normativa del C.S.I.C. dio solución a ciertas situaciones problemáticas en materia de ordenación, como por ejemplo ubicar al final de cada periodo - año, siglo, etc.- los documentos con fecha incompleta, los que no poseen datación o los que fueran conjeturables; sistema que posteriormente ha sido aceptado por los responsables de las normativas que figuran en nuestras colecciones diplomáticas más sobresalientes ", así como por la misma Comisión Internacional de Diplomática ${ }^{12}$.

Otro tipo de problemas relacionados con la organización y ordenación de los documentos en las colecciones diplomáticas, como por ejemplo el de los documentos falsos, los documentos perdidos, los "vidimus", los documentos insertos, los documentos mencionados en pancartas y las copias auténticas, han sido objeto de atención de la Comisión Internacional de Diplomática ${ }^{13}$ y alguna de sus soluciones han sido aceptadas para casuísticas similares en las normativas españolas ${ }^{14}$.

\section{Estructura de la edición y contenido de sus partes}

Las normativas españolas analizadas coinciden generalmente en un modelo de edición de documentos o estructura general configurada por las partes y en el orden siguiente:

- Fecha.

- Resumen.

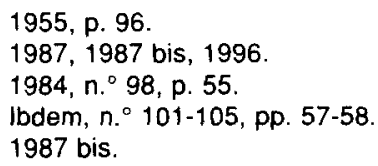


- Aparato crítico.

- Texto.

- Anotaciones históricas y disertación crítica.

Como se podrá ver, esta estructura, en todo similar a la recomendada por la Comisión Internacional de Diplomática salvo en el orden de colocación del texto, presenta variantes en nuestras normativas en lo que se refiere a:

a) La terminología de las diferentes partes.

b) El contenido de alguna de éstas.

c) La disposición de las anotaciones históricas y la disertación crítica.

Solamente las normativas de los profesores Z. García Villada y F. Arribas Arranz difieren del resto, puesto que su estructura básica se fundamenta, respectivamente, en el siguiente orden:

- Resumen, remitente y destinatario, fecha, texto, aparato críticonotas sobre las fuentes, citas de autor ${ }^{15}$.

- Resumen, fecha, aparato crítico, texto, anotaciones históricas y disertación crítica ${ }^{16}$.

\subsection{La datación o fecha}

La datación o fecha de un documento es la indicación del lugar y tiempo de un acontecimiento; la fórmula de datación comporta en consecuencia una fecha crónica y una fecha tópica ${ }^{17}$.

Las normativas españolas analizadas no ofrecen posturas unívocas en sus directrices para expresar la datación de los documentos, como veremos seguidamente tras el estudio comparativo de los diferentes aspectos que la conforman.

\section{a) Elementos de la data crónica y su expresión}

En cuanto a la consideración de cuáles han de ser los elementos que definen la data crónica, sí se ha observado una postura común, puesto

\footnotetext{
$151921,5^{\circ}$, p. 304.

$16 \quad 1965$, vid. edición de sus láminas pp. 22 y ss.

17 Vocabulaire International de la Diplomatique. Ed. M. ${ }^{a}$ Milagros Cárcel Ortí. Universitat de Valencia, 1997, (2. ${ }^{2}$ ed.), n. ${ }^{\circ}$ 560-563.
} 
que todos los autores convienen en utilizar el año, el mes y el día expresados en el cómputo actual, coincidiendo en este aspecto con la normativa dictada por la Comisión Internacional de Diplomática.

No obstante los profesores E. Sáez y C. Sáez aceptan la posibilidad de completar estos datos básicos con otros, como la feria semanal, la hora, etc. cuando la información del documento lo permita, lo cual aparece totalmente desaconsejado en la normativa internacional ${ }^{18}$.

Asimismo F. Arribas Arranz se aleja en este aspecto de la normativa más generalizada para el caso de la datación de los documentos latinos fechados, pues aconseja mantener la datación romana, acompañándola, eso sí, de su equivalencia al cómputo actual entre paréntesis.

\section{b) Elementos de la data tópica y su expresión}

También existe una coincidencia expresa sobre la determinación de esta parte de la datación, que aludirá al término donde tiene lugar el negocio u otorgamiento, expresado en ortografía moderna, esto es, al uso actual.

La utilización del término entre comillas, aconsejado por la Comisión Internacional de Diplomática, para los casos en que el lugar no haya sido identificado, ya ha sido incorporada en las normativas españolas más recientes ${ }^{19}$.

\section{c) Ordenación de los elementos de la datación}

Si en la definición de los elementos de la datación se ha podido comprobar una aparente uniformidad, no ocurre lo mismo en la expresión de la fórmula de datación del documento, es decir, en el orden de agrupamiento de la fecha crónica y la tópica.

Mientras algunos autores se muestran partidarios de establecer el orden: lugar, día, mes y año ${ }^{20}$, otros prefieren el correspondiente al año, mes, dia y lugar, siguiendo en ello la postura de la Comisión Internacional de Diplomática que, al justificar este planteamiento, apela al

181984, n..$^{\circ} 13$, p. 26.

$1996,2.2 .1 .$, p. 30.

1921, 1955, 1983, 1965. 
criterio de ordenación cronológica que debe regir las colecciones diplomáticas ${ }^{21}$.

\section{d) Dificultades técnicas}

De todos los problemas que puede generar el establecimiento de la datación de un documento, algunas normativas españolas han fijado su atención sobre uno en particular. Nos referimos a aquellos documentos que, no presentando información explícita en torno a sus fechas crónica y tópica, permiten ser datados a través de otras vías de análisis, siendo unánime la postura de que sean suplidos o rectificados, según la situación, utilizando el signo crítico [ ] o ¿ ? para los casos dudosos ${ }^{22}$.

Si a pesar de todo esta labor resultara imposible, también habrá que destacarlo, recurriendo a signos críticos cuya aceptación, sin embargo, no aparece uniformada. Pensemos por ejemplo en los documentos sin fecha crónica; todas las normativas españolas utilizan el signo (s.f.) mientras que la Comisión Internacional de Diplomática aconseja (s.d.); para los documentos sin fecha tópica, el signo español utilizado es (s.l.) y la normativa internacional recomienda no indicarlo salvo que esta ausencia sea netamente significativa.

\subsection{El resumen}

El planteamiento que rige la elaboración del resumen en las distintas normativas presenta diferencias acusadas en lo concerniente a tres aspectos básicos.

\section{a) Terminología}

"Registro o resumen de contenido" ${ }^{23}$ "extracto» ${ }^{24}$, "regesto" ${ }^{25}$, "regesto o resumen" ${ }^{26}$ constituyen los términos más difundidos entre las normativas españolas.

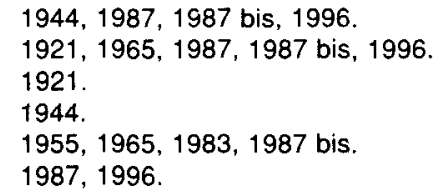


Siguiendo una vez más a la Comisión Internacional de Diplomática observamos la existencia de un término distinto, «análisis diplomático" o simplemente "análisis", cuyo significado no es otro que el relativo a un breve resumen del documento en el que se sintetiza su contenido histórico o jurídico ${ }^{27}$. De esta manera queda claramente diferenciado del término «regesta» o "catálogo", que alude al conjunto de análisis procedentes de una misma cancillería, ordenados cronológicamente ${ }^{28}$.

\section{b) Redacción y estilo}

Las propuestas elaboradas para configurar el resumen reparan sobre dos cuestiones bien distintas: la naturaleza del mismo -exhaustividad, concisión-y el estilo - directo, indirecto-.

Son más los autores que proponen la elaboración de resúmenes precisos y concisos, con contenidos breves aunque completos en los que siempre figure el otorgante, el destinatario y una síntesis del negocio histórico o jurídico tratado ${ }^{29}$.

No obstante, también se observa la tendencia inversa, en la que se proponen resúmenes que constituyan un fiel reflejo del contenido informativo del documento y en la que se puedan introducir además cuantos añadidos aclaratorios se estimen oportunos e incluso las conjeturas elaboradas para resolver determinadas dificultades ${ }^{30}$.

En cuanto al estilo de redacción sólo las normativas provinientes de los organismos profesionales ofrecen recomendaciones expresas, declarándose partidarias del estilo directo ${ }^{31} \mathrm{o}$ de ambos, según convenga ${ }^{32}$.

c) Utilización de nombres de personas y lugares

El análisis de este aspecto permite observar una postura común, abiertamente decidida por lograr el máximo de claridad, pues todas las normativas coinciden en utilizar los nombres propios de persona y lugar con sus

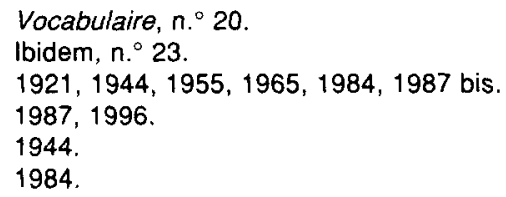


equivalentes actuales en el caso de que éstos lo tuvieran. Incluso algunas proponen incluir cuantos datos complementarios sean precisos para realizar las aclaraciones oportunas; asi por ejemplo, en la cita de nombres de reyes convendrá añadir el ordinal que faltare, especificar su reinado, etc.

Si la identificación no fuera posible, todas las normativas aceptan recoger el nombre tal como figura en el documento. No obstante, las diferencias surgen en el procedimiento y, por ello, en los signos críticos empleados: entre comillas y la aclaración entre corchetes ${ }^{33}$, o bien el término original acompañado de la propuesta entre paréntesis ${ }^{34}$.

\subsection{Aparato crítico}

El aparato crítico y las notas, que corresponden como ya se vió anteriormente a la tercera y quinta partes de la estructura general de la edición de un documento, registran el mayor grado de diferencia en las normativas elaboradas para tal fin. Por lo que respecta a la parte que ahora nos ocupa -el aparato crítico-, dichas diferencias se centran en cuestiones relacionadas con la terminología asignada y con el planteamiento que preside su contenido básico.

\section{a) Terminología}

“Notas sobre las fuentes" ${ }^{35}$, «aparato erudito" ${ }^{36}$, "descripción del documento" "37, "aparato crítico" ${ }^{38}$ son los términos empleados para designar esta parte que la Comisión Internacional de Diplomática define como "aquella parte de la edición de un documento en la que se mencionan y describen las distintas fuentes de conocimiento que se tienen sobre el mismo (original, copias, minuta, extracto, etc.), así como todas las referencias bibliográficas de que haya sido objeto ${ }^{39}$. Su objetivo, en consecuencia, no es otro que establecer la tradición documental del documento editado, razón por la que la Comisión empleó el vocablo francés "tableau de la tradition».

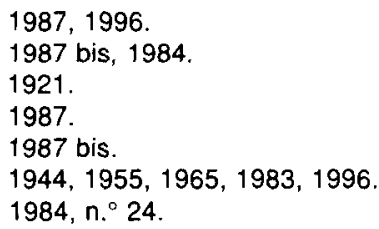




\section{b) Contenido básico}

Ahora bien, el análisis comparativo de las distintas normativas españolas ha permitido diferenciar dos corrientes o tendencias cuyos intereses resultan bien definidos a la hora de abordar el contenido del aparato crítico, respondiendo en esencia, por un lado, a la que podría denominarse «corriente paleográfica» $y$, por el otro, a la "corriente de editores de colecciones diplomáticas".

Si analizamos en primer lugar las directrices propuestas en las normativas aparecidas en los diferentes tratados y manuales de Paleografía, rápidamente se observa una total despreocupación por determinar la tradición documental o el grado de ingenuidad del documento editado ${ }^{40}$. Esta parte de la edición queda resuelta con la inclusión de una doble información: por una parte, la procedencia del documento, es decir, el depósito donde se conserva y su signatura, y por la otra, la relación de referencias bibliográficas existentes sobre el mismo en los casos en que el documento no fuera inédito. Algunos autores incluyen también la identificación del tipo de letra ${ }^{41}$, mientras que otros ni la mencionan por dedicarle posteriormente, en la parte destinada a notas, un importante comentario paleográfico ${ }^{42}$. Pero ninguno de los analizados considera oportuno introducir una descripción de los caracteres externos del documento, a excepción de la existencia de ilustraciones gráficas. Como se tendrá ocasión de ver más adelante, a pesar de la escasa consideración prestada a esta parte de la edición, la que hemos venido en denominar como corriente paleográfica centra su mayor interés en la transcripción del texto y en el capítulo destinado a notas.

Frente a las normativas elaboradas por dicha corriente paleográfica, las planteadas por los editores de colecciones diplomáticas insisten en la importancia concedida al aparato crítico, razón que, en cualquier caso, tampoco les lleva a una deseada uniformidad de criterios ${ }^{43}$.

En primer lugar $y$ desde el punto de vista del contenido que debe ofrecer esta parte, las normativas englobadas en la citada corriente coinciden en establecer la tradición documental, recogiendo para ello tanto el original como todo género de copias conservadas, determinando además la naturaleza de éstas y su dependencia o vinculación; igualmente se registran las referencias

$1921,1955,1965,1983$.

1965.

$1955,1983$.

1944, 1984, 1987, 1987 bis, 1996. 
bibliográficas sobre el documento editado. No obstante, existen autores que completan esta información con un apartado más destinado a comentar la problemática del documento, incluyendo aclaraciones relacionadas con su cronología y autenticidad ${ }^{44}$, cuestiones ambas que la Comisión Internacional de Diplomática incluye dentro de la disertación crítica.

En segundo lugar tampoco se ha encontrado una postura común en el tema relacionado con la descripción física de las fuentes, existiendo partidarios de la exhaustividad ${ }^{45} \mathrm{y}$, por contra, defensores de evitar las descripciones prolijas ${ }^{46}$. En este sentido, quizá sea la normativa internacional la responsable de la mejor sistematización descriptiva, centrada en los siguientes elementos de comentario: naturaleza del soporte y formato en caso de no ser éste último habitual; dimensiones; sistema de sellado; estado de conservación; depósito y signatura; notas dorsales o marginales y en los documentos partidos, señalar el modo de partición.y la divisa.

Asimismo, las discrepancias son manifiestas en los referente al nivel de descripción del original y las copias, con determinadas normativas que propugnan mantener idéntica atención a uno y otras ${ }^{47}$, otras que consideran necesario marcar una diferencia, reduciendo al mínimo la descripción física de las copias ${ }^{48} y$, finalmente, algunas que descartan completamente la descripción de éstas últimas ${ }^{49}$.

En tercer y último lugar también se ha podido detectar un marcado alejamiento en el procedimiento de elaboración del repertorio de referencias bibliográficas, con autores que propugnan la inclusión de todo tipo de ediciones, traducciones, regestos, facsímiles, comentarios e, incluso, el establecimiento de la dependencia de su utilización ${ }^{50}$, y otros que son partidarios de incluir todas las ediciones y una selección muy concreta de regestos ${ }^{51}$, quedando esta postura más cercana a la de la Comisión Internacional, que plantea la necesidad de recoger, siempre, cualquier edición anterior, mientras que los extractos, traducciones, facsímiles y fotografias, inventarios de archivos, catálogos de regesta y otras obras diversas sólo se considerarán cuando aporten algún elemento sustantivo a la tradición documental.

$45 \quad 1984,1987,1996$.

461944,1987 bis.

47 $1987,1996$.

481984.

$49 \quad 1987$ bis.

$50 \quad 1987,1996$.

$51 \quad 1987$ bis. 
Finalmente no convendría olvidar que, en cuanto a la forma de cita de estas referencias bibliográficas, la Comisión Internacional de Diplomática se ha mostrado decididamente partidaria del sistema francés ${ }^{52}$.

\subsection{Notas históricas y disertación crítica}

Todas las normativas españolas convienen en disponer la quinta y última parte de la edición a continuación de la transcripción del texto, a manera de notas al pié de la misma, ubicándola en consecuencia de manera distinta a la aconsejada en la normativa internacional, es decir, a continuación del aparato crítico ${ }^{53}$.

El contenido asignado a esta parte difiere según las distintas normativas. La inmensa mayoría de los autores, y entre ellos los editores de colecciones diplomáticas, coinciden en reservar un primer apartado para las variantes de palabras muy excepcionales que figuran en las distintas fuentes del documento y que contribuyen a lograr el establecimiento del texto ${ }^{54}$; algunos amplían este estudio a las variantes de lectura realizadas por los autores de las ediciones del documento ${ }^{55}$.

En segundo término, existe una preocupación bastante difundida por registrar todas aquellas particularidades o circunstancias que hayan afectado a la grafía - tachaduras, interpolaciones, interlineados, raspados, espacios en blanco, correcciones, agregados, etc.-y al soporte -lagunas, pérdidas, roturas, mordeduras de tinta, etc. $-{ }^{56}$.

En tercer y último lugar también hay autores partidarios de añadir un cuerpo de anotaciones históricas, a manera de aclaraciones que hagan más inteligible el uso del texto, facilitando la identificación de topónimos, personajes citados, acontecimientos, alusión o citas de fuentes, etc. ${ }^{57}$.

El contenido que A. Millares dedica a esta parte de la edición merece un comentario particular, pues frente a lo que se acaba de ver, la edición de sus documentos presenta en su lugar un minucioso y exhaustivo comentario paleográfico y diplomático, analizando en el primero el tipo de escritura, las abreviaturas y los nexos, y la tipología documental en el segundo.

\footnotetext{
$1984, n \cdot 36$

$1984, n .^{\circ} 95$

$1921,1944,1984,1987,1987$ bis, 1996.

1987,1987 bis, 1996.

1944, 1955, 1965, 1983, 1984, 1987, 1987 bis, 1996.

$1921,1944,1984,1987,1996$.
} 
Para finalizar, no queremos obviar las diferencias encontradas en materia de terminología. Para algunos autores el estudio de las variantes, anteriormente mencionado, y las anotaciones sobre particularidades del soporte y la grafía constituyen el «aparato crítico» de sus ediciones ${ }^{58}$; en otras normativas se diferencia el estudio de variantes, al que se sigue denominando "aparato crítico», de las "notas o citas de autor", con las que se alude a las aclaraciones históricas ${ }^{59}$; en normativas asociadas con tratados y manuales de Paleografía se habla de "observaciones al texto", reuniendo bajo este epigrafe solamente las especiales circunstancias que afectan a la grafía ${ }^{60}$. Ante tal diversidad de acepciones, otros autores han optado por la sencillez, denominando a esta parte de la edición «notas al pie de la transcripción» ${ }^{61}$.

\section{El texto: normas para su transcripción}

Todas las normativas españolas disponen de un conjunto variable de directrices para realizar la transcripción del texto de los documentos, si bien no todas coinciden en los mismos problemas técnicos ni, naturalmente, aportan idénticas soluciones.

Con objeto de sistematizar el análisis comparativo de dichas soluciones, conviene señalar previamente que dichas normas abordan una doble problemática: en primer lugar, asuntos y aspectos relacionados con la disposición y presentación del texto - alineaciones, sistemas de indicación de líneas, columnas y folios, suscripciones y signos-, y en segundo lugar una amplia casuística relativa a cuestiones de grafía, abreviaturas y ortografía.

Veamos a continuación las principales convergencias y discrepancias que ofrecen nuestras normativas en los asuntos mencionados.

\subsection{Disposición y presentación del texto}

\section{a) Alineaciones}

La separación artificial de párrafos para facilitar la comprensión del texto constituye una práctica temprana y plenamente difundida en las nor-

\footnotetext{
$581944,1987,1987$ bis.

1921,1944

$1955,1965$.

1996.
} 
mativas españolas. Sin embargo, no todos los autores coinciden a la hora de establecer el carácter de tales separaciones, existiendo normativas que lo dejan a juicio del editor ${ }^{62}$, otras que son partidarias de diferenciar exclusivamente la fecha y las suscripciones aunque dando cabida a otro género de separaciones para documentos de gran extensión y de carácter muy excepcional ${ }^{63}$, y finalmente las que, siguiendo las recomendaciones de la Comisión Internacional de Diplomática, establecen los párrafos según el esquema del discurso diplomático: protocolo inicial, preámbulo, exposición y dispositivo, cláusulas finales, suscripciones, signos y fecha.

La indicación de esta práctica a través del uso de un signo crítico sólo encontró aceptación en la normativa del profesor Arribas Arranz, quien empleó el símbolo $=$.

b) Indicación de líneas, columnas, páginas y folios

Todas las normativas son partidarias de reflejar en la edición el cambio de línea o columna del texto transcrito, asi como la numeración de las mismas; igualmente, cuando el texto procede de un códice diplomático habrá que indicar el cambio de folio o, en su caso, de página. No obstante, los sistemas seguidos para señalar tales indicaciones son los que no resultan coincidentes.

En la edición de documentos originales, se ha propuesto señalar el cambio de línea mediante una línea vertical ${ }^{64} \mathrm{u}$ oblicua ${ }^{65}$, pareciendo apta en otras normativas cualquiera de ambas ${ }^{66}$. Además, también ha parecido conveniente la numeración de todas las líneas del texto ${ }^{67}$, o el recurso de numerarlas de tres en tres ${ }^{68}$ o de cinco en cinco ${ }^{69}$, existiendo normativas que proponen cualquiera de los sistemas anteriores ${ }^{70}$.

En la edición de documentos procedentes de cartularios, registros, tumbos o de cualquier tipo de códice diplomático, será preciso indicar el cambio y número de folio, así como si éste es recto o verso, el de página y, en

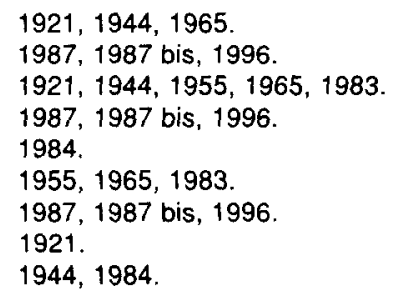


su caso, la columna, proponiéndose para estas últimas la numeración ordinal arábiga o las primeras letras del alfabeto en minúscula.

\section{c) Suscripciones y signos}

En todas las normativas españolas se puede observar una orientación idéntica para abordar la transcripción de suscripciones y cualquier tipo de elementos figurados: signos, rúbricas, monogramas y crismón.

En cuanto a las primeras, cuando se trate de las correspondientes a confirmantes o testigos, se exige el respeto al orden y disposición en que aparezcan en el original. Respecto a los elementos figurados todas las normativas aconsejan reflejarlos en el edición mediante la indicación del elemento correspondiente expresado en cursiva y encerrado entre paréntesis o corchetes. La transcripción del monograma constantiniano ha sido aceptada bajo una doble forma: (crismón ) o (Christus).

\subsection{Grafías, abreviaturas y ortografía}

\section{a) Grafías}

El respeto a la grafía original del documento constituye un principio general sobre el que se asientan todas las normativas de transcripción, las cuales, asimismo, aceptan la corrección de errores o faltas evidentes en nota, nunca en el texto editado. Solamente la Comisión Internacional de Diplomática prevee la posibilidad de incluir tales correcciones en la edición del texto cuando el documento sea de una época en la que la lengua disponga de unos formularios plenamente fijados o normalizados, no pudiendo obviar tampoco en estos casos su advertencia y explicación en una nota ${ }^{71}$.

Ahora bien, este principio general no significa que las transcripciones deban convertirse en una reproducción facsimilar del texto original, puesto que su finalidad básica es contribuir a hacerlo más inteligible. Para ello, las distintas normativas contemplaron tempranamente una serie de directrices que permitieran afrontar la transcripción de un conjunto de grafías concretas propias de la lengua latina y de textos romances: ç, i vocal, r/s

$71 \quad 1984$, n. 59 
largas, $u / v, y$, consonantes dobles al inicio de palabra, $R$ en medio de palabra, los dos tipos de ese con valor zeta, uso de la h, signos de la copulativa y.

Igualmente, nuestras normativas recogen un conjunto de recomendaciones indicativas y signos críticos, no siempre coincidentes, que permiten señalar en la transcripción la existencia de frecuentes y habituales problemas técnicos, tales como:

- Lecturas dudosas: al término conjeturable le acompañará el signo (?) y una aclaración en nota ${ }^{72}$.

- Palabras con errores evidentes: al término le acompañará el signo (sic) ${ }^{73}$.

- Existencia de lagunas: en caso de que no puedan ser suplidas, unos autores proponen el signo ${ }^{*} . .{ }^{*} 74$, otros el signo $(\ldots)^{75}$ y finalmiente otros $[\ldots]$ 76; pero si pudieran ser restituidas se podrá indicar con el signo [ [ ${ }^{\pi}$.

- Tachaduras, repeticiones y otras anomalías: indicarlas en nota, nunca en el texto editado ${ }^{78}$.

- Letras o palabras sobrantes: utilizar el signo $<>79$.

- Omisiones, errores: el mismo signo anterior $<>80$.

- Adiciones e interlineados: algunos autores proponen no utilizar ningún signo, aclarándolo en nota ${ }^{81}$, otros utilizan el signo / ${ }^{82}$.

- Espacios en blanco: unas normativas son partidarias de utilizar el signo [...] $]^{83}$ y otras [en blanco] ${ }^{84}$.

\section{b) Abreviaturas}

Todas las normativas españolas aceptan como principio general la resolución o desarrollo de las abreviaturas que presentan los textos origi-

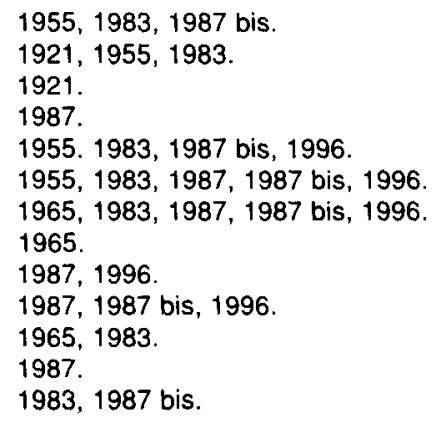


nales, si bien unas creen conveniente indicar en la edición la parte restituida utilizando letras cursivas o el paréntesis ${ }^{85} \mathrm{y}$ otras son partidarias de no indicarlo ${ }^{86}$.

La postura más generalizada ante el caso de las abreviaturas no deducibles o conjeturables es la de abordar su resolución apoyándose en otros casos que presente el propio documento y en su defecto en otros de la misma época. Para diferenciar estos casos particulares de aquellos cuya resolución se encuentra más o menos regularizada, algunos autores han propuesto su diferenciación mediante el empleo de letra cursiva ${ }^{87}$.

Por lo demás todas las normativas mantienen idénticas soluciones para el desarrollo de abreviaturas caracteristicas, como las de origen griego (Xptus. y sus derivados), Dno., las formas de la copulativa "y», la numeración romana, etc.

\section{c) Ortografía}

Todas las normativas coinciden en separar o unir palabras, sílabas o letras que aparezcan incorrectas en el documento, siguiendo los principios de la ortografía clásica. Ante el caso concreto de las contracciones, la postura también resulta unánime, pues todas plantean la necesidad de respetarlas salvo en los casos en que dieran lugar a interpretaciones erróneas.

También se ha podido constatar una gran unanimidad ante el uso de mayúsculas y minúsculas, pues todas las normativas abogan por su transcripción según el uso actual. Algunas en particular recogen una amplia casuística relacionada con el uso de mayúsculas en textos latinos, coincidiendo netamente con la presentada al respecto por la Comisión Internacional de Diplomática ${ }^{88}$.

Finalmente, se ha podido apreciar una absoluta coincidencia en el planteamiento y utilización de los signos de puntuación, ante los cuales, todas las normativas defienden un uso actualizado aunque con moderación. Además de la coma, el punto y coma, el punto seguido o aparte, los dos puntos y el paréntesis, se aprueba la utilización de los signos de interrogación y de admiración; también se recomienda el uso de las comillas

\footnotetext{
$1921,1955,1965,1983$.

$1944,1984,1987,1987$ bis, 1996.

$1987,1996$.

1984, 1987, 1987 bis, 1996.
} 
para destacar palabras textuales atribuidas a una persona y el empleo de la letra cursiva para las citas literales de algún texto, acompañadas de la correspondiente identificación en nota ${ }^{89}$. Existe por último un acuerdo expreso en no acentuar la edición de textos romances, salvo en los casos en que el acento sirva para diferenciar vocablos.

\section{Presentación de la edición: cuerpo y tamaño de la letra}

La presentación material de las colecciones diplomáticas, aunque aparentemente pueda resultar un tema irrelevante, ha constituido asimismo objeto de interés en las normativas elaboradas para la edición y transcripción de documentos. No cabe la menor duda que un análisis comparativo sobre este punto resultaría trivial. Sin embargo no lo es tanto la comprobación de una constante preocupación por diferenciar las distintas partes de la estructura del documento a través del uso de tipos y cuerpos de letra diversos, consiguiendo de esta manera una división rápida y eficaz entre la fecha, el resumen, el aparato crítico, el texto y las notas. La Comisión Internacional de Diplomática ha recomendado en este sentido distinguir el resumen, el aparato crítico y el texto, utilizando la letra cursiva para el primero y un cuerpo de letra inferior al empleado en el texto para el segundo.

Sin pretender la exposición de las diferencias apreciadas en este aspecto dentro de las normativas españolas, si podemos afirmar que la propuesta realizada por la Comisión Internacional ya estaba presente en la redactada en 1944 por la Escuela de Estudios Medievales, habiendo ejercido su influencia en todas las posteriores.

\section{Apéndices e índices}

Solamente las normativas creadas para la transcripción y edición de colecciones diplomáticas dedican parte de su atención a procurar unas directrices generales que presidan la confección de apéndices e índices, resultando suficientemente generalistas como para no entrar nunca en discrepancias relevantes ${ }^{90}$.

La elaboración de un apéndice general formado a partir de los encabezamientos de la edición — fecha y resumen- parece presidir la mayoría

1944, 1984, 1987, 1987 bis, 1996. 
de las colecciones diplomáticas, así como la realización de un apéndice de correspondencias cuando éste sea necesario (orden de un cartulario/ordenamiento en la edición).

Por lo que respecta a los índices, todos los autores convienen en la necesidad de configurar tres: onomástico, toponímico y materias, existiendo partidarios de fundirlos en un índice alfabético ${ }^{91}$. La casuística que preside cada uno de éstos ha quedado perfectamente sistematizada por la Comisión Internacional de Diplomática. 